\title{
RETRACTION
}

\section{Apoptosis Induced by the Histone Deacetylase Inhibitor FR901228 in Human T-Cell Leukemia Virus Type 1-Infected T-Cell Lines and Primary Adult T-Cell Leukemia Cells}

Naoki Mori, Takehiro Matsuda, Masayuki Tadano, Takao Kinjo, Yasuaki Yamada, Kunihiro Tsukasaki, Shuichi Ikeda, Yoshihiro Yamasaki, Yuetsu Tanaka, Takao Ohta, Teruo Iwamasa, Masao Tomonaga, and Naoki Yamamoto

Division of Molecular Virology and Oncology, Graduate School of Medicine, Divisions of Child Health and Welfare, Pathology and Cell Biology, and Immunology, Faculty of Medicine, University of the Ryukyus, Nishihara, Okinawa 903-0215, Division of Laboratory Medicine, Nagasaki University Graduate School of Biomedical Sciences, Nagasaki 852-8501, Department of

Hematology, Molecular Medicine Unit, Atomic Bomb Disease Institute, Nagasaki University School of Medicine, Nagasaki 852-8523, Department of Hematology, Sasebo City General Hospital, Sasebo 857-8511, Department of Internal Medicine, Kokura Memorial Hospital, Kitakyushu 802-8555, and

Department of Molecular Virology, Bio-Response, Graduate School, Tokyo Medical and Dental University, Tokyo 113-8519, Japan

Volume 78, no. 9, p. 4582-4590, 2004. The publisher hereby retracts the above article due to evidence of data manipulation, a clear violation of ASM's ethical standards. 